Pacific Journal of Mathematics

POLYNOMIAL EQUATIONS OF IMMERSED SURFACES 


\title{
POLYNOMIAL EQUATIONS OF IMMERSED SURFACES
}

\author{
S. Akbulut AND H. KING
}

\begin{abstract}
If $V$ is a nonsingular real algebraic set we say $H_{t}\left(V ; \mathbf{Z}_{2}\right)$ is algebraic if it is generated by nonsingular algebraic subsets of $V$.

Let $V^{3}$ be a 3-dimensional nonsingular real algebraic set. Then, we prove that any immersed surface in $V^{3}$ can be isotoped to an algebraic subset if and only if $H_{i}\left(V: \mathbf{Z}_{2}\right) i=1,2$ are algebraic. This isotopy above carries the natural stratification of the immersed surface to the algebraic stratification of the algebraic set. Along the way we prove that if $V$ is any nonsingular algebraic set then any simple closed curve in $V$ is $\varepsilon$-isotopic to a nonsingular algebraic curve if and only if $H_{1}\left(V: \mathbf{Z}_{2}\right)$ is algebraic.
\end{abstract}

Let $V^{3}$ be a 3-dimensional nonsingular real algebraic set. We call a homology group of $V$ algebraic if it is generated by nonsingular algebraic subsets. In this paper we prove:

THEOREM. The following are equivalent:

(a) If $f: M^{2} a \rightarrow V^{3}$ is any immersion of a closed smooth surface in general position, then $f\left(M^{2}\right)$ is isotopic to an algebraic subset $Z$ of $V^{3}$ by an arbitrarily small isotopy. This isotopy carries the natural stratification of $f\left(M^{2}\right)$ to the algebraic stratification of $Z$.

(b) $H_{1}\left(V ; \mathbf{Z}_{2}\right)$ and $H_{2}\left(V ; \mathbf{Z}_{2}\right)$ are algebraic.

To be more precise for $i=1,2$ let $A H_{i}\left(V^{3} ; \mathbf{Z}_{2}\right)$ be the subgroup of $H_{i}\left(V^{3} ; \mathbf{Z}_{2}\right)$ generated by nonsingular algebraic subsets. Then $H_{i}\left(V ; \mathbf{Z}_{2}\right)$ is algebraic if it is equal to $A H_{i}\left(V ; \mathbf{Z}_{2}\right)$. In particular zero homology groups are algebraic. We will refer to elements of $A H_{i}\left(V^{3} ; \mathbf{Z}_{2}\right)$ as algebraic homology classes. This definition is consistent with the conventions of $\left[\mathrm{AK}_{1}\right]$.

In case $f$ is an imbedding this theorem reduces to a special case of Proposition 1 below, which is Theorem 4.1 and Remark 4.2 of [AK $\mathbf{A K}_{1}$ ]. Recall, if $W^{n}$ is a nonsingular algebraic set of dimension $n$, then $A H_{n-1}\left(W ; \mathbf{Z}_{2}\right)$ is the subgroup of $H_{n-1}\left(W ; \mathbf{Z}_{2}\right)$ generated by nonsingular algebraic subsets. Also if $M \subset W$ is a closed submanifold, denote the 
$\mathbf{Z}_{2}$-homology class in $W$ induced by the fundamental class of $M$ by $[M]_{2}$. Then

Proposition 1. A codimension one closed smooth submanifold $M$ of $W$ is $\varepsilon$-isotopic to a nonsingular real algebraic subset if and only if $[M]_{2} \in$ $A H_{n-1}\left(W ; \mathbf{Z}_{2}\right)$. Furthermore, this isotopy can fix any smooth submanifold $L$ of $M$ which is already a nonsingular algebraic set.

Remark. Proposition 1 remains true if $L$ is a union of nonsingular algebraic sets in $M([\mathrm{~T}])$.

We first prove a codimension two version of this proposition for $V^{3}$, which is an interesting result in itself.

Proposition 2. A simple closed curve $C \subset V^{3}$ is $\varepsilon$-isotopic to a nonsingular algebraic curve if and only if $[C]_{2} \in A H_{1}\left(V ; \mathbf{Z}_{2}\right)$. Furthermore this isotopy can fix any collection of points in $C$.

REMARK. This proposition remains true if $V^{3}$ is replaced by a nonsingular algebraic set of any dimension. The proof is essentially the same.

LEMMA 3. Let $C \subset V^{3}$ be a nonsingular algebraic curve and $L \subset V^{3}$ be a smooth manifold. Then $C$ can be moved by an $\varepsilon$-isotopy to a nonsingular algebraic curve $C^{\prime}$ which is transversal to $L$.

Proof. Let $F^{2}$ be the boundary of a small closed tubular neighborhood of $C$ in $V . F$ is a circle bundle over $C$ and hence has a section, so after a small isotopy of $F$ we can assume that $C \subset F$. Since $F$ is null homologous, by Proposition 1 , it is $\varepsilon$-isotopic to a nonsingular algebraic surface $Z$ with $C \subset Z$. By the terminology of $\left[\mathbf{A K}_{\mathbf{1}}\right] C$ is a stable algebraic set. Stable algebraic sets have the required property (Proposition 4.3 of $\left.\left[\mathbf{A K}_{1}\right]\right)$.

LEMMA 4. If $V^{3}$ is orientable and $F^{2} \subset V^{3}$ is a compact orientable surface with $\partial F^{2}=C \cup A$ where $A$ is a nonsingular algebraic curve, then $C$ is $\varepsilon$-isotopic to a nonsingular algebraic curve.

Proof. Since $V$ is orientable $F$ has a trivial normal bundle in $V$. Let $F^{\prime}=\partial(F \times I) \subset V^{3}$ corners smoothed, and $C \cup A=\partial(F \times 0) \subset F^{\prime}$. $C \cup A$ separates $F^{\prime}$. Since $\left[F^{\prime}\right]_{2}=0$ by Proposition $1 F^{\prime}$ is $\varepsilon$-isotopic to a nonsingular algebraic surface $Z$ with $A \subset Z$. After a small isotopy of $C$ 
we can assume $C \subset Z$. Then $C \cup A$ separates $Z$; this means $[C]_{2}=[A]_{2}$ $\in A H_{1}\left(Z ; \mathbf{Z}_{2}\right)$. Hence by Proposition $1 C$ is $\varepsilon$-isotopic to a nonsingular algebraic curve $C^{*}$ in $Z . C^{*}$ is the required algebraic curve.

REMARK. We can assume that the isotopy $C \leadsto C^{*}$ fixes any finite number of points of $C$. This is because by Proposition 1 we can arrange that $Z$ and $C^{*}$ fix these points.

LEMMA 5. If $S \subset V^{3}$ is an orientable surface and

$$
i_{*}: H_{1}\left(V-S ; \mathbf{Z}_{2}\right) \rightarrow H_{1}\left(V ; \mathbf{Z}_{2}\right)
$$

is the map induced by the inclusion, then $\operatorname{ker}\left(i_{*}\right) \subset A H_{1}\left(V-S ; \mathbf{Z}_{2}\right)$.

Proof. From the homology exact sequence

$$
H_{2}\left(V, V-S ; \mathbf{Z}_{2}\right) \stackrel{\partial}{\rightarrow} H_{1}\left(V-S ; \mathbf{Z}_{2}\right) \stackrel{i_{*}}{\rightarrow} H_{1}\left(V ; \mathbf{Z}_{2}\right) \quad \operatorname{im}(\partial)=\operatorname{ker}\left(i_{*}\right) .
$$

Also we have isomorphisms

$$
H_{2}\left(V, V-S ; \mathbf{Z}_{2}\right) \stackrel{\text { excision }}{\leftrightarrows} H_{2}\left(N, \partial N ; \mathbf{Z}_{2}\right) \stackrel{\text { Thom }}{\cong} H_{1}\left(S ; \mathbf{Z}_{2}\right)
$$

where $N$ is a small closed tubular neighborhood of $S$ in $V$. In particular $N$ is an $I$-bundle over $S$, and $\partial N$ is an $\dot{I}$-bundle over $S\left(\dot{I}=S^{0}\right)$. From the above isomorphism we see that elements of $\operatorname{im}(\partial)$ are represented by the induced $\dot{I}$-bundles $\tilde{\gamma}$ over the curves $\gamma$ of $S$
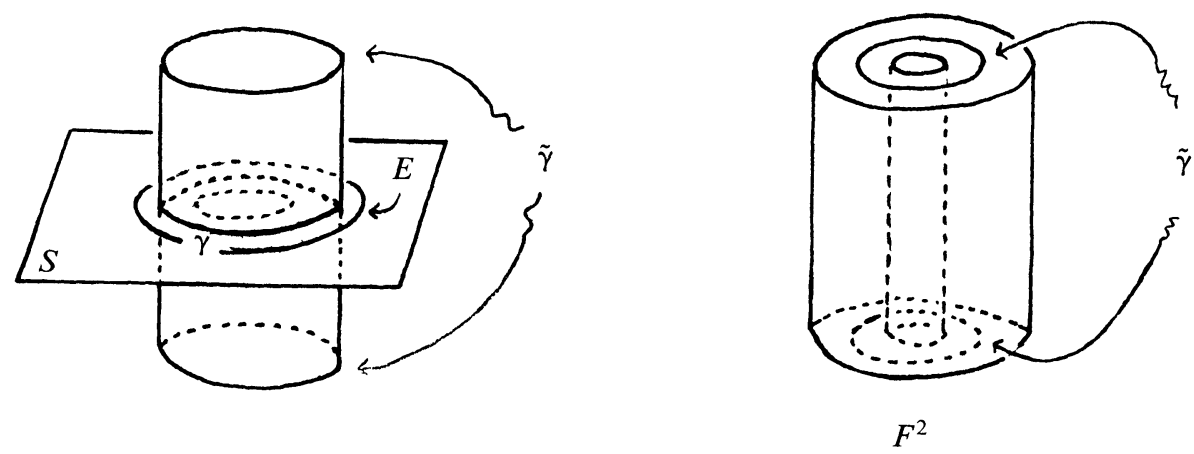

Let $E$ be a small closed tubular neighborhood of $\gamma$ in $S$, since $S$ orientable $E \approx \gamma \times I$. Let $E^{\prime}$ be the induced $I$-bundle over $E$. Let $F^{2}=\partial E^{\prime}$. Clearly $F^{2}$ is a null homologous surface in $V$ containing $\tilde{\gamma}$. Furthermore $\tilde{\gamma}$ separates $F^{2}$. By Proposition $1 F^{2}$ can be $\varepsilon$-isotoped to a 
nonsingular algebraic surface $Z$. After a small isotopy of $\tilde{\gamma}$ we can assume that $\tilde{\gamma} \subset Z$. Since $\tilde{\gamma}$ separates $Z$, by Proposition $1 \tilde{\gamma}$ is $\varepsilon$-isotopic to a nonsingular algebraic curve $\gamma^{*}$ in $Z$. By construction $\gamma^{*} \subset V-S$ and $[\tilde{\gamma}]_{2}=\left[\gamma^{*}\right]_{2} \in A H_{1}\left(V-S ; \mathbf{Z}_{2}\right)$.

LemMA 6. Every element of $A H_{1}\left(V ; \mathbf{Z}_{2}\right)$ can be represented by a connected nonsingular algebraic curve.

Proof. Let $\alpha \in A H_{1}\left(V ; \mathbf{Z}_{2}\right)$ then $\alpha$ is represented by a union of nonsingular algebraic curves $C=C_{1} \cup \cdots \cup C_{k}$. By Lemma 3 we can assume that they are disjoint. Let $S$ be the boundary of a closed tubular neighborhood of $C$. Since the normal bundle of $C$ has nowhere zero section, after an $\varepsilon$-isotopy of $S$ we can assume that $C \subset S$. Then by tubing the components of $S$ we get a connected surface $S^{\prime}$ with $C \subset S^{\prime}$. Let $C_{i}^{\prime}$ be $\varepsilon$-isotopic copies of $C_{i}$ on $S^{\prime}$ which are in general position with $C_{i}$. Connect $C_{i}^{\prime}, i=1, \ldots, k$, by tubes in $S^{\prime}$ to get a connected curve $C^{\prime}=C_{1}^{\prime} \# \cdots C_{k}^{\prime}$ such that $C^{\prime}$ is homologous to $C$ in $S^{\prime}$

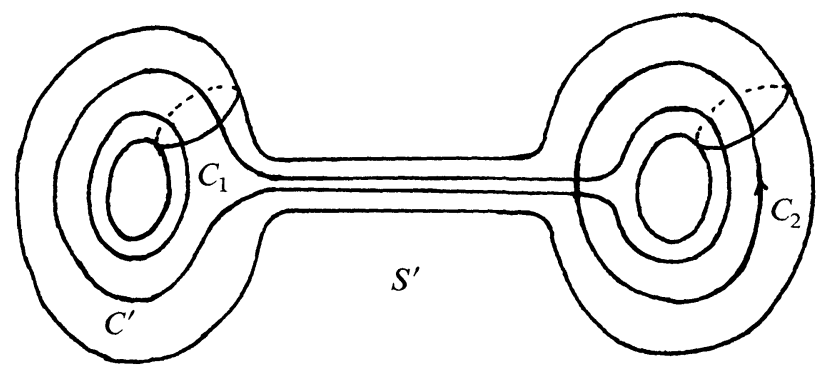

By construction $\left[S^{\prime}\right]_{2}=0$ in $H_{2}\left(V ; Z_{2}\right)$, so by Proposition 1 we can $\varepsilon$-isotop $S^{\prime}$ to a nonsingular algebraic surface $Z$ with $C \subset Z$. Continue to denote the isotopic copy of $C^{\prime}$ in $Z$ by $C^{\prime}$. Again since $\left[C^{\prime}\right]_{2}=[C]_{2} \in$ $A H_{1}\left(Z ; \mathbf{Z}_{2}\right)$ by Proposition $1, C^{\prime}$ is $\varepsilon$-isotopic to a nonsingular algebraic curve $C^{*}$ in $Z$. $C^{*}$ is connected and $\alpha=[C]_{2}=\left[C^{*}\right]_{2} \in A H_{1}\left(V ; \mathbf{Z}_{2}\right)$.

Proof of Proposition 2. We will prove this in three steps,

Case $1 . V^{3}$ is orientable.

Let $c=[C] \in H_{1}(V ; \mathbf{Z})$. Since $[C]_{2}$ is algebraic there is a nonsingular algebraic curve $A \subset V$ such that $[C]=[A]+2 b$ for some $b \in H_{1}(V ; \mathbf{Z})$. This means if $B \subset V$ is a simple closed curve with $b=[B]$, then $A \cup 2 B$ $\cup C$ bounds an orientable surface. Here $2 B$ denotes the link $B \cup B^{\prime}$ where $B^{\prime}$ is a parallel copy of $B$, so $2 B$ is a boundary of an orientable surface $B \times I$ in $V$. By Lemma 4 we can assume that $2 B$ is a nonsingular algebraic curve. Again by Lemma $4 C$ is $\varepsilon$-isotopic to a nonsingular 
algebraic curve. By the Remark following Lemma 4 we can assume that this isotopy fixes any finite number of points of $C$.

\section{Case 2. $[C]_{2}=0$ in $H_{1}\left(V ; \mathbf{Z}_{2}\right)$}

Let $S \subset V$ be a surface representing the dual of the first Steifel-Whitney class $w_{1}(V)$ of $V$. We can assume that $C \cap S=\varnothing$. This is because by homological reasons $C \cap S$ must be an even number of points, and we can modify $S$ as in the picture below without affecting its homology class.

old $S$
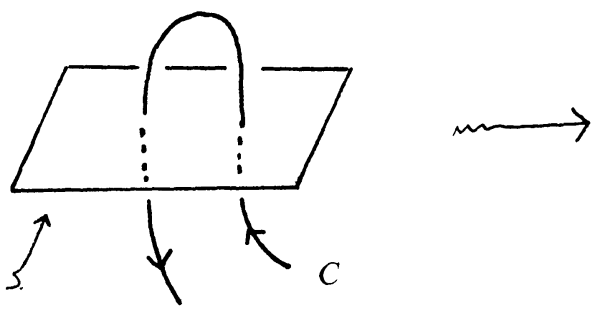

new $S$

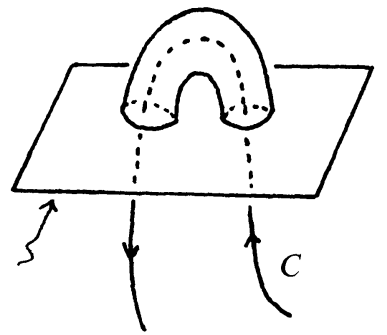

Hence $C \subset V-S$, and by assumption $[C]_{2} \in \operatorname{ker}\left(i_{*}\right)$ where

$$
i_{*}: H_{2}\left(V-S ; \mathbf{Z}_{2}\right) \rightarrow H_{2}\left(V ; \mathbf{Z}_{2}\right)
$$

is the induced map by inclusion. Since $[S]_{2}=w_{1}(V), S$ is orientable (exercise), so by Lemma $5[C]_{2} \in A H_{1}\left(V-S ; \mathbf{Z}_{2}\right)$. Since $V-S$ is orientable, by Case $1 C$ is $\varepsilon$-isotopic to a nonsingular algebraic curve in $V-S$, fixing any finite number of points of $C$.

Case 3. The general case.

We choose a connected nonsingular algebraic curve $D$ disjoint from $C$ so that $[C]_{2}=[D]_{2}$. Let $S$ be the boundary of a closed tubular neighborhood of $C \cup D$. As in the proof of Lemma 6 after a small isotopy of $S$ we can assume that $C \cup D \subset S$, and let $S^{\prime}$ be the connected surface obtained by tubing the two components of $S$. By construction $C \cup D \subset S^{\prime}$. Let $C^{\prime}$ and $D^{\prime}$ be $\varepsilon$-isotopic transverse copies of $C$ and $D$ in $S^{\prime}$. Then by tubing $C^{\prime}$ and $D^{\prime}$ in $S^{\prime}$ we get a curve $E=C^{\prime} \# D^{\prime}$ as in the picture

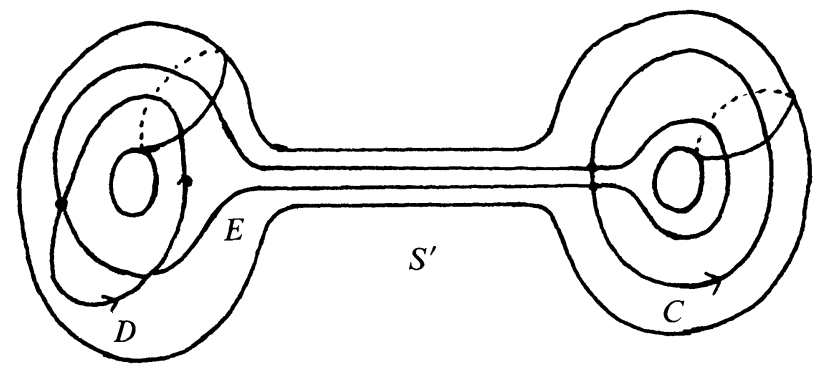


By construction we have

(a) $\left[S^{\prime}\right]_{2}=0$ in $H_{2}\left(V ; \mathbf{Z}_{2}\right)$

(b) $[E]_{2}=[C \cup D]_{2}$ in $H_{1}\left(S^{\prime} ; \mathbf{Z}_{2}\right)$

(c) $[E]_{2}=0$ in $H_{1}\left(V ; \mathbf{Z}_{2}\right)$

By Case $2 E$ is $\varepsilon$-isotopic to a nonsingular algebraic curve $E^{*}$ in $V$ fixing the points $E \cap(C \cup D)$. After an $\varepsilon$-isotopy of $S^{\prime}$ we may assume $C \cup D \cup E^{*} \subset S^{\prime}$. By Proposition 1 (and by the remark following it) we can $\varepsilon$-isotop $S^{\prime}$ to a nonsingular algebraic surface $Z$ with $D \cup E^{*} \subset Z$. Let $C^{\prime}$ be the corresponding $\varepsilon$-isotopic copy of $C$ in $Z$. Since $\left[C^{\prime}\right]_{2}=$ [ $\left.D \cup E^{*}\right]_{2} \in A H_{1}\left(Z ; \mathbf{Z}_{2}\right)$ by Proposition $1 . C^{\prime}$ is $\varepsilon$-isotopic to a nonsingular algebraic curve $C^{*}$ in $Z$. Furthermore given any finite number of points on $C_{1}$ by Proposition 1 we can require that all these isotopies fix these points.

Proof of the Theorem. First we show (b) $\Rightarrow$ (a). For every $y \in f\left(M^{2}\right)$ consider $n(y)=\max \left\{n \mid\right.$ there are $n$ distinct points $x_{1}, \ldots, x_{n} \in M$ with $f\left(x_{i}\right)=y$ for $\left.i=1,2, \ldots, n\right\}=$ the cardinality of $f^{-1}(y) . f(M)$ is a stratified set with strata $\left\{L_{i}\right\}_{l=1}^{3}$ where $L_{i}$ are the $i$-fold point sets, $L_{l}=\{y \in f(M) \mid n(y)=i\}$. Call $d(f)=\max \left\{i \mid L_{i} \neq \varnothing\right\}$, then $d(f) \leq$ 3 and if $d(f)=3, L_{3}$ is a collection of points (the triple points). Let $M_{3}=f^{-1}\left(L_{3}\right)$. By ([AK $\left.\mathbf{K}_{1}\right]$, Lemma 2.3) there is a unique immersion $f^{\prime}$ with $d\left(f^{\prime}\right)=2$ making the following commute

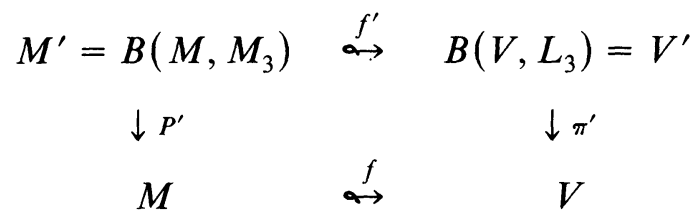

where the vertical maps are the blowing up maps along the centers $M_{3}, L_{3}$. Since the points are algebraic, we can assume that $V^{\prime} \stackrel{\pi^{\prime}}{\rightarrow} V$ is the algebraic blow up of $V$ along $L_{3}$.

Since $d\left(f^{\prime}\right)=2$ the 2 -fold point set $L_{2} \subset V^{\prime}$ of the map $f^{\prime}$ is a smooth manifold (i.e., collection of smooth circles). Let $M_{2}=\left(f^{\prime}\right)^{-1} L_{2}$. Once again by $\left[\mathbf{A K}_{\mathbf{1}}\right]$ there is a unique immersion $f^{\prime \prime}$ with $d\left(f^{\prime \prime}\right)=1$ (i.e., it is an imbedding) making the following commute

$$
\begin{aligned}
& M^{\prime \prime}=B\left(M^{\prime}, M_{2}\right) \stackrel{f^{\prime \prime}}{\leftrightarrow} \quad B\left(V^{\prime}, L_{2}\right)=V^{\prime \prime} \\
& \downarrow P^{\prime \prime} \quad \downarrow \pi^{\prime \prime} \\
& M^{\prime} \\
& \stackrel{f^{\prime}}{\leftrightarrow} \quad V^{\prime}
\end{aligned}
$$


where the vertical maps are the blowing up maps. In particular $M^{\prime \prime}=M^{\prime}$ and $p^{\prime \prime}=$ identity, since $M_{2} \subset M^{\prime}$ is codimension one.

$$
V^{\prime}=V \stackrel{k}{\# \mathbf{R} P^{3}} \text { so } H_{i}\left(V^{\prime}\right)=H_{i}(V) \oplus H_{i}\left(\# \mathbf{R} P^{3}\right) \text { for } i=1,2 \text {; in }
$$

particular $H_{1}\left(V^{\prime} ; \mathbf{Z}_{2}\right)$ and $H_{2}\left(V^{\prime} ; \mathbf{Z}_{2}\right)$ are algebraic. By Proposition 2 the curve $L_{2}$ is $\varepsilon$-isotopic to a nonsingular algebraic set. We can change $f(M)$ by a small isotopy in $V$ keeping $L_{3}$ fixed so that the corresponding double point set $L_{2}$ in $V^{\prime}$ is this nonsingular algebraic set. Therefore we can take $\pi^{\prime \prime}$ to be the algebraic blow up along $L_{2}$, in particular $V^{\prime \prime}$ is a nonsingular algebraic set.

We claim that $H_{2}\left(V^{\prime \prime} ; \mathbf{Z}_{2}\right)$ is algebraic. This can be seen by the homology exact sequences

$$
\begin{aligned}
& \cdots \quad \rightarrow \quad H_{2}\left(C^{\prime \prime}\right) \stackrel{i_{*}}{\rightarrow} \quad H_{2}\left(V^{\prime \prime}\right) \quad \rightarrow \quad H_{2}\left(V^{\prime \prime}, C^{\prime \prime}\right) \quad \rightarrow \quad \ldots \\
& \downarrow \pi_{*}^{\prime \prime} \quad \downarrow \pi_{*}^{\prime \prime} \quad \cong \downarrow \text { excision } \\
& \cdots \quad \rightarrow \quad H_{2}\left(C^{\prime}\right) \quad \rightarrow \quad H_{2}\left(V^{\prime}\right) \quad \rightarrow \quad H_{2}\left(V^{\prime}, C^{\prime}\right)
\end{aligned}
$$

where all the homology groups have coefficient $\mathbf{Z}_{2}$, and $C^{\prime}, C^{\prime \prime}$ are closed tubular neighborhoods of $L_{2},\left(\pi^{\prime \prime}\right)^{-1}\left(L_{2}\right)$ respectively. Since $\pi^{\prime \prime}$ is degree $1 \pi_{*}^{\prime \prime}$ is onto, and by the above diagram $\operatorname{ker} \pi_{*}^{\prime \prime}=\operatorname{im}\left(i_{*}\right)$ where $i$ is the inclusion $C^{\prime \prime} \hookrightarrow V^{\prime \prime}$. So $H_{2}\left(V^{\prime \prime} ; \mathbf{Z}_{2}\right)$ is generated by the nonsingular algebraic sets $\left(\pi^{\prime \prime}\right)^{-1}\left(L_{2}\right)$, and $\left(\pi^{\prime \prime}\right)^{-1}\left(S_{i}\right)$ where $S_{i}$ are surfaces in $V^{\prime}$. By Proposition 1 we can assume $S_{i}$ are nonsingular algebraic surfaces. By ([AK $\left.\mathbf{A K}_{1}\right]$ Proposition 4.3) we can assume $S_{i}$ are transverse to $L_{2}$. Hence $H_{2}\left(V^{\prime \prime} ; \mathbf{Z}_{2}\right)$ is generated by nonsingular algebraic sets.

By Proposition 1 we can $\varepsilon$-isotop the smooth submanifold $f^{\prime \prime}\left(M^{\prime \prime}\right)$ to a nonsingular algebraic subset $Q$ of $V^{\prime \prime}$ by a smooth isotopy. By ([AK $]$ Lemma 2.5) $\pi^{\prime} \circ \pi^{\prime \prime}(Q)$ is an algebraic set. $\pi^{\prime} \circ \pi^{\prime \prime}(Q)$ is isotopic to $f(M)$ by a small isotopy. More precisely, the last remark can be seen by applying $\left(\left[\mathbf{A} \mathbf{K}_{2}\right]\right.$ Proposition 5.5). Namely $\left[\mathbf{A K}_{2}\right]$ gives an isotopy $h_{t}$ : $V^{\prime \prime} \rightarrow V^{\prime \prime}$ such that

(1) $h_{0}=$ Id,

(2) $h_{1}\left(f^{\prime \prime}\left(M^{\prime \prime}\right)\right)=Q$,

(3) $h_{t}^{-1}\left(\pi^{-1}(x)\right)=\pi^{-1}(x)$ for all $x \in L \subset V$, where $\pi=\pi^{\prime} \circ \pi^{\prime \prime}, L=$ $L_{3} \cup \pi^{\prime}\left(L_{2}\right)$.

Then we can define an isotopy

$$
g_{t}: V \rightarrow V \text { by } g_{t}(x)=\pi h_{t}(y) \text { for } \begin{cases}y=\pi^{-1}(x), & \text { if } x \notin L, \\ y \in \pi^{-1}(x), & \text { if } x \in L .\end{cases}
$$


(Notice $\pi$ is a diffeomorphism over the complement of $L$.) $g_{t}$ gives an isotopy of $f(M)$ to $\pi(Q)$ fixing $L$ pointwise. Also $g_{t}$ is smooth in the complement of $L$.

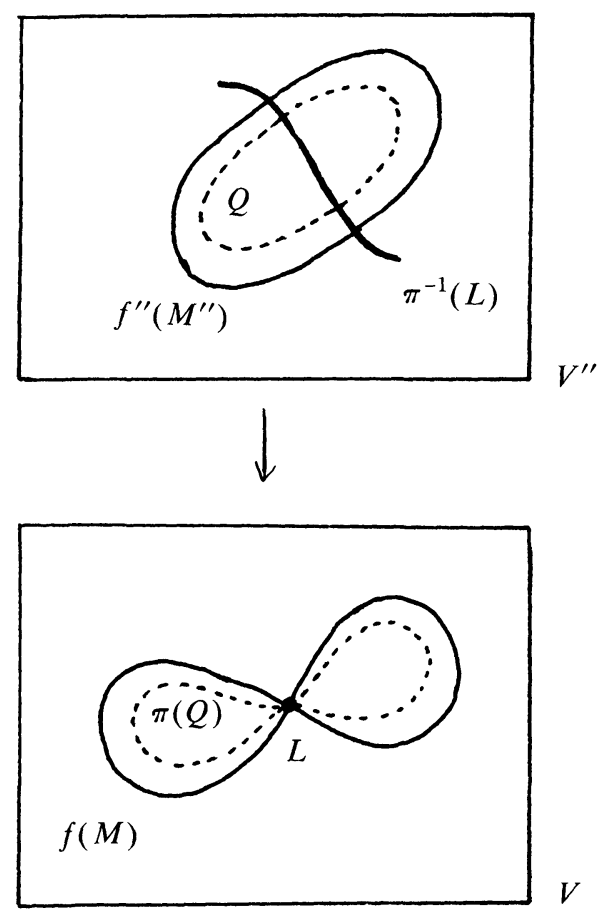

It remains to show (a) $\Rightarrow$ (b). Clearly (a) implies $H_{2}\left(V ; \mathbf{Z}_{2}\right)$ algebraic. To see $H_{1}\left(V ; \mathbf{Z}_{2}\right)$ algebraic we write every simple closed curve $C \subset V^{3}$ as the double point of an immersion. $C$ has a normal bundle $C \tilde{\times} D^{2} \subset V$. Then $C \tilde{\times} X \subset V$ where $X$ is the figure eight, so $C \tilde{\times} X=f\left(S^{1} \tilde{\times} S^{1}\right)$ where $f: S^{1} \tilde{x} S^{1} \rightarrow V$ is the obvious immersion. Hence by (a) $f\left(S^{1} \tilde{\times} S^{1}\right)$ can be made algebraic and $C$ is the singular set of this algebraic set.

Note added in proof. After writing this paper we have been informed by W. Kucharz that he had proved a special case of Proposition 2 when $V$ is orientable in "Topology of Real Algebraic Threefolds" Duke Math. Journal, vol. 53, No. 4, Dec. 1986.

\section{REFERENCES}

$\left[\mathrm{AK}_{1}\right]$ S. Akbulut and H. King, Submanifolds and homology of nonsingular real algebraic varieties, Amer. J. Math., (1985), 45-83.

$\left[\mathrm{AK}_{2}\right]$ _ Real algebraic structures on topological spaces, IHES, 53 (1981), 79-162. 
$\left[\mathrm{AK}_{3}\right]$, The topology of real algebraic sets with isolated singularities, Ann. of Math., 113 (1981), 425-446.

[T] A. Tognoli, Algebraic approximation of manifolds and spaces, Sem Bourbaki 32 éme aneé (1979) No. 548.

Received September 23, 1986 and in revised form March 3, 1987. Both authors were supported in part by NSF funds.

Michigan State University

EAST LANSING, MI 48824 - 1027

AND

UNIVERSITY OF MARYLAND

College PARK, MD 20742 



\section{PACIFIC JOURNAL OF MATHEMATICS EDITORS}

\author{
V. S. VARADARAJAN \\ (Managing Editor) \\ University of California \\ Los Angeles, CA 90024 \\ HERBERT ClEMENS \\ University of Utah \\ Salt Lake City, UT 84112 \\ R. FINN \\ Stanford University \\ Stanford, CA 94305
}

\author{
HERMANN FLASCHKA \\ University of Arizona \\ Tucson, AZ 85721
}

RAMESh A. GANGolli

University of Washington Seattle, WA 98195

VAUGHAN F. R. JONES

University of California

Berkeley, CA 94720

\author{
ROBION KIRBY \\ University of California \\ Berkeley, CA 94720
}

C. C. MOORE

University of California

Berkeley, CA 94720

HAROLD STARK

University of California, San Diego

La Jolla, CA 92093

\section{ASSOCIATE EDITORS}
R. AREnS
E. F. BECKENBACH
B. H. NEUMANN
F. WOLF
K. YOSHIDA

(1906-1982)

\section{SUPPORTING INSTITUTIONS}

\begin{abstract}
UNIVERSITY OF ARIZONA
UNIVERSITY OF BRITISH COLUMBIA

CALIFORNIA INSTITUTE OF TECHNOLOGY

UNIVERSITY OF CALIFORNIA

MONTANA STATE UNIVERSITY

UNIVERSITY OF NEVADA, RENO

NEW MEXICO STATE UNIVERSITY

OREGON STATE UNIVERSITY
\end{abstract}

\author{
UNIVERSITY OF OREGON \\ UNIVERSITY OF SOUTHERN CALIFORNIA \\ STANFORD UNIVERSITY \\ UNIVERSITY OF HAWAII \\ UNIVERSITY OF TOKYO \\ UNIVERSITY OF UTAH \\ WASHINGTON STATE UNIVERSITY \\ UNIVERSITY OF WASHINGTON
}

The Supporting Institutions listed above contribute to the cost of publication of this Journal, but they are not owners or publishers and have no responsibility for its content or policies.

Mathematical papers intended for publication in the Pacific Journal of Mathematics should be in typed form or offset-reproduced (not dittoed), double spaced with large margins. Please do not use built up fractions in the text of the manuscript. However, you may use them in the displayed equations. Underline Greek letters in red, German in green, and script in blue. The first paragraph must be capable of being used separately as a synopsis of the entire paper. In particular it should contain no bibliographic references. Please propose a heading for the odd numbered pages of less than 35 characters. Manuscripts, in triplicate, may be sent to any one of the editors. Please classify according to the scheme of Math. Reviews, Index to Vol. 39. Supply name and address of author to whom proofs should be sent. All other communications should be addressed to the managing editor, or Elaine Barth, University of California, Los Angeles, California 90024.

There are page-charges associated with articles appearing in the Pacific Journal of Mathematics. These charges are expected to be paid by the author's University, Government Agency or Company. If the author or authors do not have access to such Institutional support these charges are waived. Single authors will receive $\mathbf{5 0}$ free reprints; joint authors will receive a total of $\mathbf{1 0 0}$ free reprints. Additional copies may be obtained at cost in multiples of 50 .

The Pacific Journal of Mathematics is issued monthly as of January 1966. Regular subscription rate: $\$ 190.00$ a year (5 Vols., 10 issues). Special rate: $\$ 95.00$ a year to individual members of supporting institutions.

Subscriptions, orders for numbers issued in the last three calendar years, and changes of address should be sent to Pacific Journal of Mathematics, P.O. Box 969, Carmel Valley, CA 93924, U.S.A. Old back numbers obtainable from Kraus Periodicals Co., Route 100, Millwood, NY 10546.

The Pacific Journal of Mathematics at P.O. Box 969, Carmel Valley, CA 93924 (ISSN 0030-8730) publishes 5 volumes per year. Application to mail at Second-class postage rates is pending at Carmel Valley, California, and additional mailing offices. Postmaster: send address changes to Pacific Journal of Mathematics, P.O. Box 969, Carmel Valley, CA 93924.

PUBLISHED BY PACIFIC JOURNAL OF MATHEMATICS, A NON-PROFIT CORPORATION Copyright (C) 1988 by Pacific Journal of Mathematics 


\section{Pacific Journal of Mathematics}

\section{Vol. 131, No. 2 December, 1988}

Selman Akbulut and Henry Churchill King, Polynomial equations of immersed surfaces .................................... 209

Alberto Baider and Richard C. Churchill, The Campbell-Hausdorff group and a polar decomposition of graded algebra automorphisms ........2 219

Wayne C. Bell and John William Hagood, Separation properties and exact

Radon-Nikodým derivatives for bounded finitely additive measures . . . 237

Dennis J. Garity, James P. Henderson and David G. Wright, Menger

spaces and inverse limits ...............................249

B. Brent Gordon, Algebraically defined subspaces in the cohomology of a

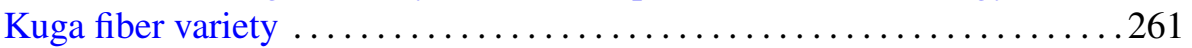

Jeffrey A. Hogan, Weighted norm inequalities for the Fourier transform on connected locally compact groups ........................... 277

Guojun Liao, A study of regularity problem of harmonic maps ..........291

Chin-pi Lu, Modules satisfying ACC on a certain type of colons ......... 303

Kunio Murasugi, Jones polynomials of periodic links

Hans Schoutens, Approximation properties for some non-Noetherian local

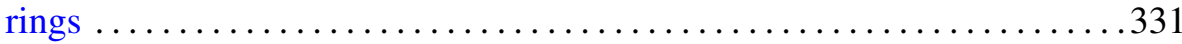

Peter Sjögren, Convergence for the square root of the Poisson kernel ...... 361 Alexandru Ion Suciu, The oriented homotopy type of spun 3-manifolds .393 